\title{
Surface Energy Band and Electron Affinity of Highly Phosphorous-doped Epitaxial CVD Diamond*
}

\author{
S. $\operatorname{Kono}^{\dagger}$ \\ Institute of Multidisciplinary Research for Advanced Materials, \\ Tohoku University, Sendai 980-8577 Japan, and \\ CREST, JST, Tokyo 150-0011, Japan
}

\author{
K. Mizuochi, G. Takyo, N. I. Plusnin, ${ }^{\ddagger}$ T. Aoyama, ${ }^{\S}$ T. Goto, and T. Abukawa \\ Institute of Multidisciplinary Research for Advanced Materials, Tohoku University, Sendai 980-8577 Japan
}

A. Namba, Y. Nishibayashi, and T. Imai

Sumitomo Electric Industry Ltd., Itami, Hyogo 664-0016, Japan

(Received 25 November 2006; Accepted 5 January 2007; Published 3 February 2007)

\begin{abstract}
The surface energy band diagrams and the electron affinity of hydrogen-terminated and oxygen-terminated highly phosphorous-doped single crystal diamond (111) surfaces have been studied by ultraviolet photoelectron spectroscopy, secondary electron spectroscopy, X-ray photoelectron spectroscopy and photoemission electron microspectroscopy. A hydrogen-terminated boron-doped diamond (001) surface was used as a reference of surface energy band diagram. The electron affinity of the H-terminated heavily P-doped diamond was determined to be $0.2 \pm 0.15$ $\mathrm{eV}$, thus close to zero. The electron affinity of the O-terminated highly P-doped diamond was determined to be $0.0 \pm 0.15 \mathrm{eV}$, thus can be negative. However, the surface energy bands for the two highly P-doped samples were found to have large amounts $(3 \mathrm{eV})$ of upward bending toward surface. [DOI: 10.1380/ejssnt.2007.33]
\end{abstract}

Keywords: Diamond; Ultraviolet photoelectron spectroscopy; Secondary electron spectroscopy; X-ray photoelectron spectroscopy; Photoemission electron micro-spectroscopy; Surface energy band diagram; Electron affinity

\section{INTRODUCTION}

Fabrication of both $p$-type and n-type diamonds is a prerequisite for the use of diamond devices such as ultraviolet emission diodes [1]. $p$-type diamonds can be synthesized rather easily by chemical vapor deposition (CVD) by boron doping. However, $n$-type diamonds are difficult to synthesize. So far the best quality $n$-type diamonds are synthesized in CVD by phosphorous doping [2, 3]. However, the donor level of interstitial phosphor in diamond is deep $(\sim 0.6 \mathrm{eV})$ below the conduction band minimum and the resistivity of phosphorous-doped $n$-type diamonds is still too high with a typical value at room temperature (RT) of $\sim 10^{5} \Omega \mathrm{cm}$.

$n$-type diamonds are also attractive in terms of diamond field emitters. In $n$-type diamonds, main carriers are electrons in the conduction band so that the electrons may easily come out through surface barrier of possible negative electron affinity (NEA) surface of CVD diamond. It is noted that the conductivity increases as phosphorous concentration increases although the carrier mobility decreases [4]. Recently, highly phosphorous-doped diamonds were synthesized that showed a low resistivity of $\sim 80 \Omega \mathrm{cm}$ at RT and the $n$-type conductivity above $\sim 400$ $\mathrm{K}[5]$. Field emission properties of an emitter array made

*This paper was presented at The 7th Russia-Japan Seminar on Semiconductor Surfaces (RJSSS-7), Vladivostok, Russia, 18-20 September, 2006.

${ }^{\dagger}$ Corresponding author: kono@tagen.tohoku.ac.jp

$\ddagger$ On leave of absence from Institute of Automation and Control Processes, Far Eastern Branch, Russian Academy of Science, Vladivostok 690041, Russia

$\S$ Present Address: Steel Research Laboratory., JFE Steel Corporation, Kawasaki, 210-0855 Japan from a similar highly phosphorous doped diamonds also showed an emission current density of $\sim 6.5 \mathrm{~mA} / \mathrm{mm}^{2}$ at a field of $\sim 18 \mathrm{~V} / \mu \mathrm{m}[6]$. This field emission efficiency is better than that of boron-doped $p$-type diamond emitter array by three orders of magnitude [6].

As described above, highly phosphorous-doped diamonds are expected to be good field emitters with electron carriers in the conduction band. Field emission is strongly affected by the energy band diagram near the surface and by the electron affinity, thus it is important to understand the surface energy band and the electron affinity in order to fabricate a good field emitter. In this work, highly phosphorous-doped single crystal diamond samples have been studied by ultraviolet photoelectron spectroscopy (UPS), secondary electron spectroscopy (SES), X-ray photoelectron spectroscopy (XPS) and photo-emission electron micro-spectroscopy (PEEM) to elucidate the surface energy band diagrams and electron affinities.

\section{EXPERIMENTAL}

Two ultrahigh vacuum (UHV) apparatuses were used. One was an electron spectrometer (VG CLAM4) equipped with a 9-channeltron electron detector, a He I/II resonance UV source, a twin-anode X-ray source $(\mathrm{Mg}, \mathrm{Al})$ and LEED optics. In order to probe a limited sample area in XPS and SES, a collimator was placed at the front end of the twin-anode X-ray source so that an area of $\sim 1.0$ $\mathrm{mm}$ diameter was irradiated by either $\mathrm{Mg} \mathrm{K} \mathrm{K}_{\alpha}$ or $\mathrm{Al} \mathrm{K}_{\alpha}$ line. A collimator was also placed at the front end of the He I/II resonance source but an area of $\sim 1.5$ (width) $\times 2$ (hight) $\mathrm{mm}^{2}$ was irradiated by $\mathrm{He} \mathrm{I} / \mathrm{II}$ resonance light. Extra deflector electrodes were placed near the sample to introduce low kinetic energy secondary electrons into the 


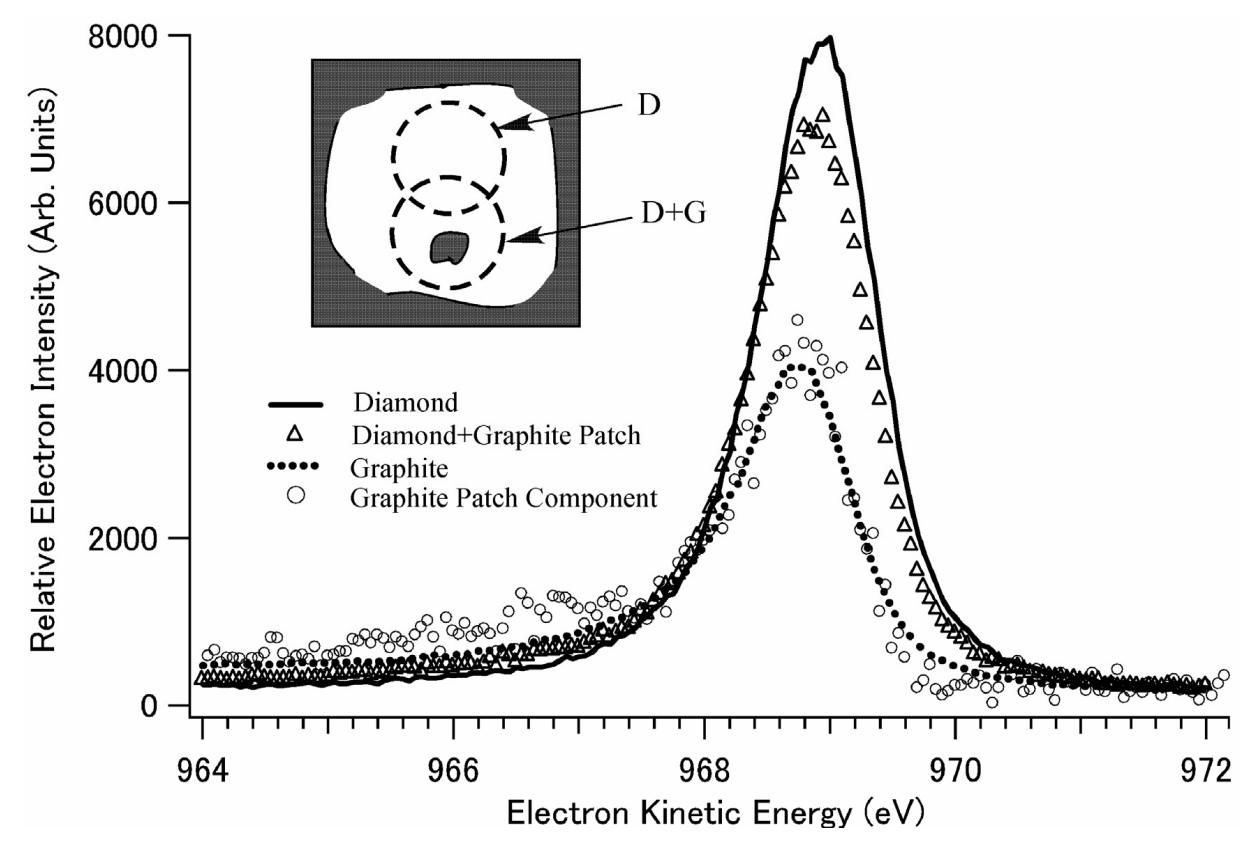

FIG. 1: C 1s XPS spectra for the standard sample of $\mathrm{B}-\mathrm{C}(001) \mathrm{H}$ and the reference graphite. Inset shows schematic illustration of the sample and two positions of X-ray beam areas.

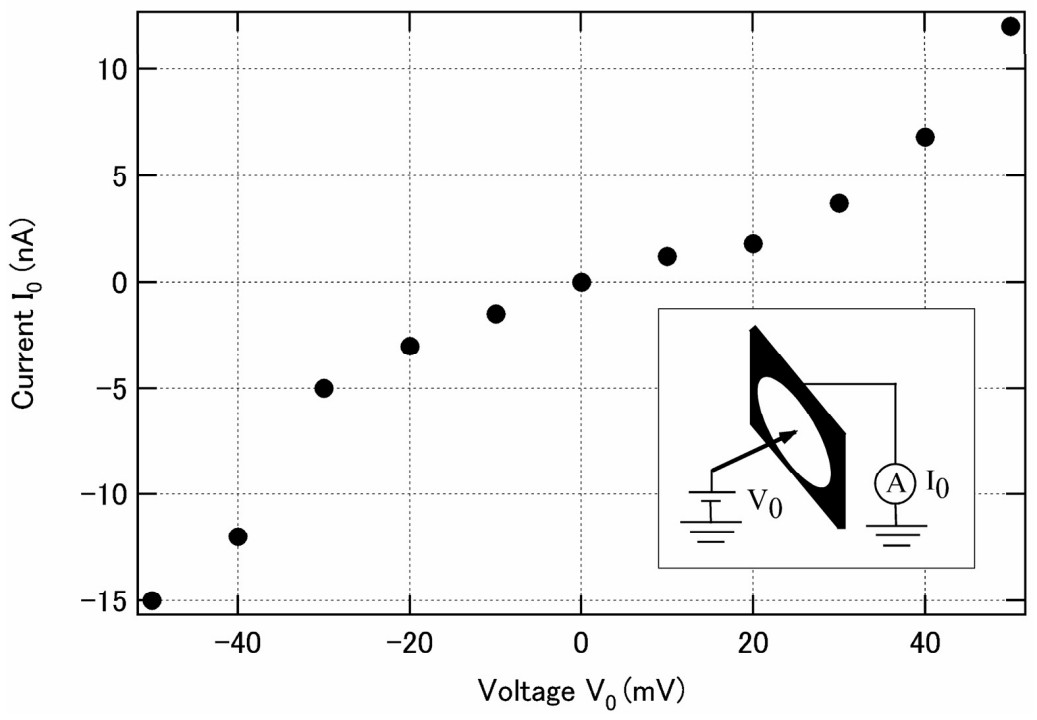

FIG. 2: Current vs. voltage curve measured in-situ on the hP-C(111)O sample. The inset shows schematic illustration of the measurement.

electron spectrometer for SES.

The other apparatus was a photoemission microspectrometer (Omicron IS-PEEM), equipped with electrostatic electron optics and a simulated hemispherical electron spectrometer. A Hg-lamp was used for excitation. The maximum photon energy from the Hg-lamp was $\sim 5 \mathrm{eV}$ but was sufficiently high for photoelectron excitation from defect levels of the diamond samples. The spatial resolution of the IS-PEEM as a photoelectron microscope was $\sim 20 \mathrm{~nm}$. The energy of secondary electrons could be analyzed from a limited area (as small as $\sim 1$ $\mu \mathrm{m}$ diameter) of the sample by adjusting the size of an iris aperture of the IS-PEEM.

The highly phosphorous-doped sample was made by epitaxial CVD growth of diamond on a $2 \times 2 \mathrm{~mm}^{2}$ HPHT $\mathrm{Ib}(111)$ substrate in a NIRIM-type MWCVD growth chamber. Source gases were $\mathrm{H}_{2}, \mathrm{CH}_{4}$ and $\mathrm{PH}_{3}$ with a $\mathrm{PH}_{3} / \mathrm{CH}_{4}$ ratio of $20 \%$. The as-grown sample surface is known to be terminated with hydrogen. This as-grown sample is called $\mathrm{hP}-\mathrm{C}(111) \mathrm{H}$ in this paper. The sample was then heated in air at $300^{\circ} \mathrm{C}$ for 1 hour, which caused oxygen terminated surface as checked by XPS. This sample is called hP-C(111)O in this paper. Electronic properties of samples made in a similar way have been reported recently [5], which showed a relatively low resistivity $(\sim 80 \Omega \mathrm{cm})$ at $300 \mathrm{~K}$ and $n$-type conduction at 400-700 K. As a standard of diamond surface energy band, a boron-doped $p$-type CVD diamond (001) was made in 

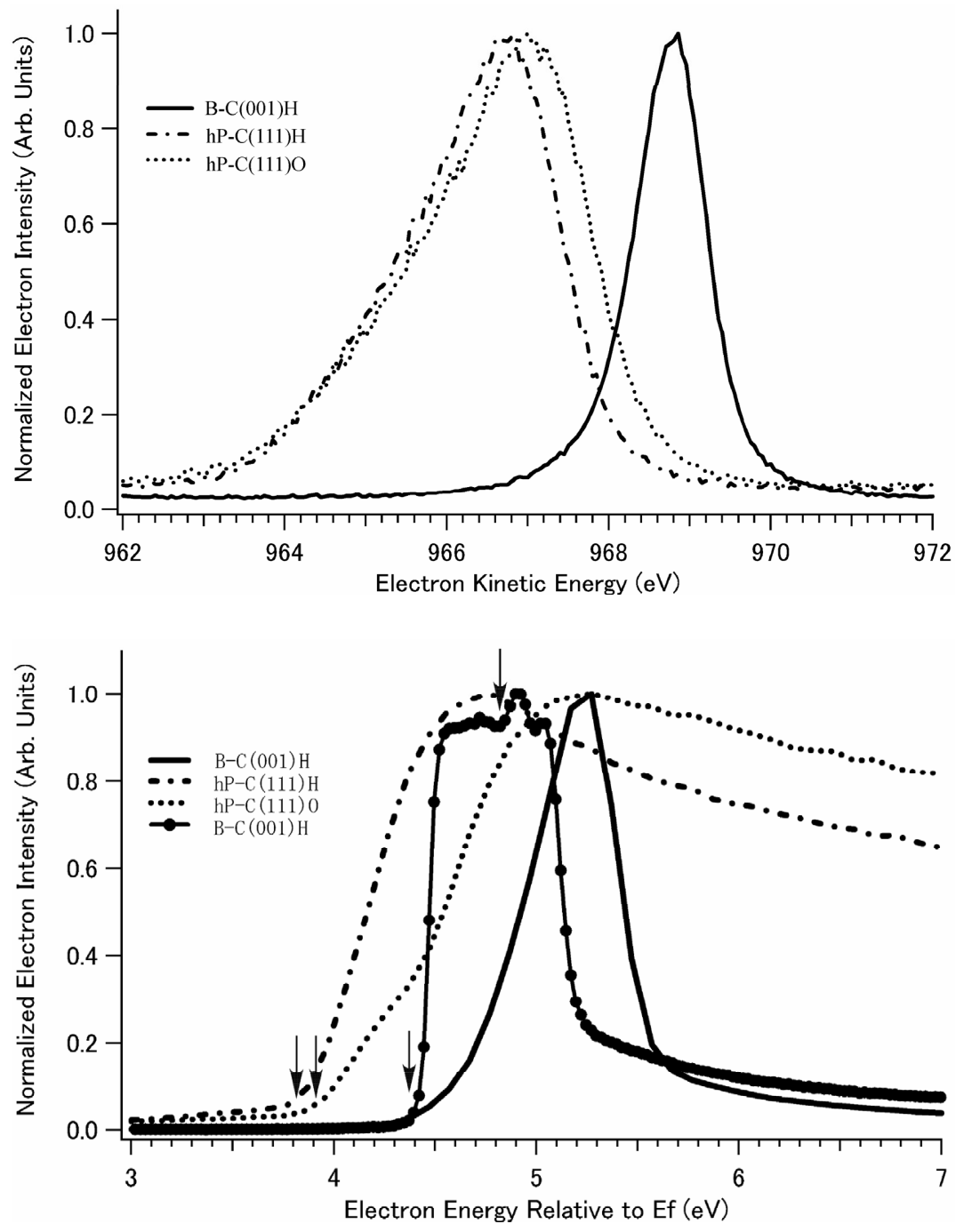

FIG. 3: Comparison of intensitynormalized $\mathrm{C} 1 s$ XPS peaks among the samples of $\mathrm{B}-\mathrm{C}(001) \mathrm{H}, \mathrm{hP}-\mathrm{C}(111) \mathrm{H}$ and $\mathrm{hP}-\mathrm{C}(111) \mathrm{O}$.
FIG. 4: Comparison of intensitynormalized SES spectra among the samples of $\mathrm{B}-\mathrm{C}(001) \mathrm{H}, \mathrm{hP}-\mathrm{C}(111) \mathrm{H}$ and $\mathrm{hP}-\mathrm{C}(111) \mathrm{O}$. Solid curve with large dots is SES spectrum as excited by $\mathrm{He}$ resonance lamp for $\mathrm{B}-\mathrm{C}(001) \mathrm{H}$ and others are those excited by $\mathrm{Mg} \mathrm{K}_{\alpha}$ $\mathrm{X}$-ray source. The electron energy is with reference to the Fermi level as determined from an $E_{A}$ peak of the graphite sample [8]. a different MWCVD growth chamber on a $3 \times 3 \mathrm{~mm}^{2}$ HPHT diamond (001) wafer. This sample is called B$\mathrm{C}(001) \mathrm{H}$ in this paper. The conditions of CVD were as follows: $\mathrm{CH}_{4} / \mathrm{H}_{2}, 0.1 \%$ (46 mbar, $400 \mathrm{sccm}$ ); $\mathrm{MW}$ power, $750 \mathrm{~W}$; substrate temperature, $750^{\circ} \mathrm{C}$; growth time, 12 h. Boron doping was not intentionally attempted but resulted due to a residual $\mathrm{B}_{2} \mathrm{H}_{6}$ gas used in a preceding heavy boron-doping CVD growth. The $\mathrm{B}$ concentration was estimated to be $\sim 1 \times 10^{18} / \mathrm{cm}^{3}$ from a sheet resistance measurement of another $\mathrm{B}-\mathrm{C}(001) \mathrm{H}$ sample made in the same batch of CVD growth. The sheet resistance measurement was performed in a similar manner as in Ref.[7]. It was noted that the sheet resistance of the second $\mathrm{B}-\mathrm{C}(001) \mathrm{H}$ sample as loaded in the CLAM4 chamber was $\sim 1 \mathrm{M} \Omega$ /square and that after annealing in vacuum at $650^{\circ} \mathrm{C}$ was $\sim 1.9 \mathrm{M} \Omega /$ square. This indicates the presence of a surface conductive layer before the in-vacuum annealing. It is usually noted that the surface conductive layer effectively vanishes after in-vacuum annealing at $650^{\circ} \mathrm{C}[7]$.

For the installation of the samples to the UHV apparatuses, the samples were held by either Ta or Mo sample holder and the periphery of the sample surface was covered by colloidal graphite paste (Aquadag) in order to establish electric contact to the sample holder. For the standard sample of $\mathrm{B}-\mathrm{C}(001) \mathrm{H}$, an isolated extra graphite patch $(\sim 0.4 \mathrm{~mm}$ diameter $)$ was pasted near the center part of the sample surface (cf. Inset of Fig. 1). This is to determine the junction properties between $\mathrm{B}-\mathrm{C}(001) \mathrm{H}$ surface and colloidal graphite paste as described later. In order to study the junction properties between the P-doped sample surface and colloidal graphite, we performed an $I-V$ test as described later. Colloidal graphite pasted on a different holder place near the sample was used for the determination of Fermi-level position of the sample holder [8]. X-ray excited SES spectra were measured in the CLAM4 chamber for the three samples with a negatively sample biased of $-10 \mathrm{~V}$. Because the light spot of the He resonance source was large, He I/II excited SES spectrum was measured for only the sample $\mathrm{B}-\mathrm{C}(001) \mathrm{H}$ at a negative sample bias of $-4.0 \mathrm{~V}$. Hg-lamp excited microSES spectra were measured in the IS-PEEM chamber at a negative bias of $-7.0 \mathrm{~V}$. For the determination of the Fermi level of SES spectra in the IS-PEEM chamber, a $4 \mathrm{kV}$ electron beam was used to excite secondary electrons from a colloidal graphite sample. X-ray photoelectrons were detected along near surface normal direction in CLAM4 spectrometer, thus XPS spectra were most bulk-sensitive. This is to probe the surface energy band as deep as possible. The detection angle for SES spectra was not well characterized since the sample bias voltage was applied. No charging-up of the samples by X-rays or 


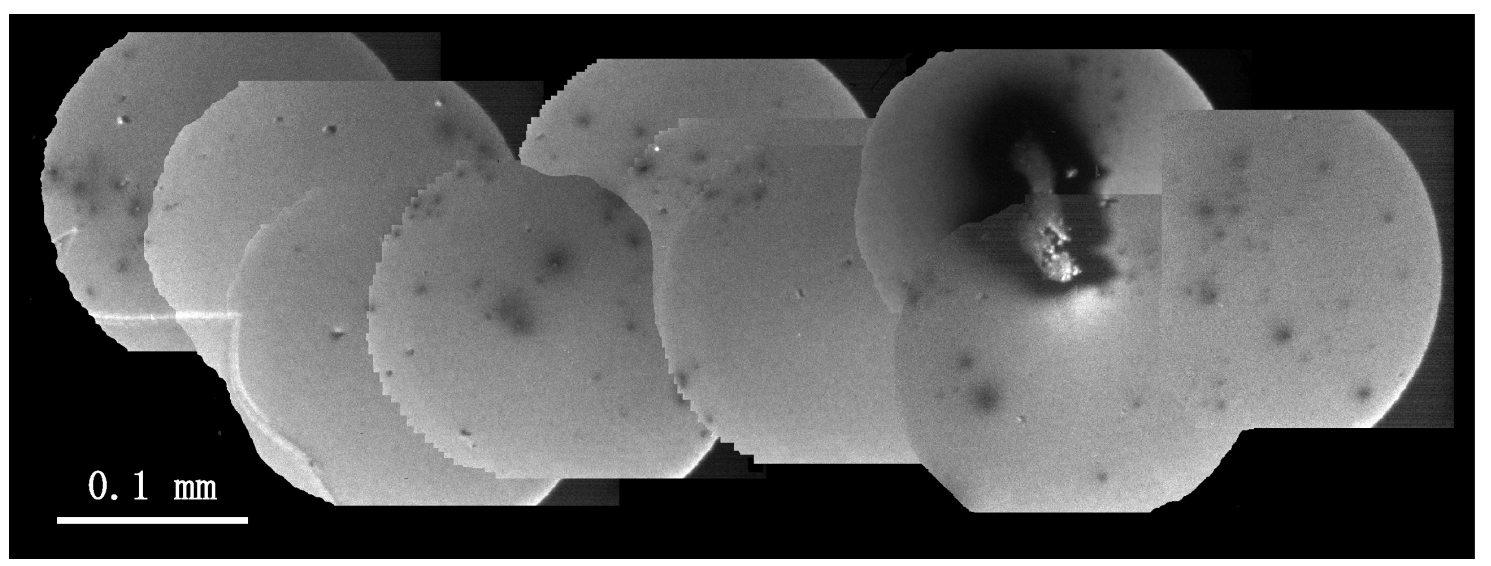

FIG. 5: A combined PEEM image of the sample hP-C(111)O as excited by the Hg-lamp.

UV-sources was noticed as no energy shift was found by changing the power of X-rays or UV-sources.

\section{EXPERIMENTAL RESULTS}

\section{A. Junction properties between diamond surface} and graphite electrode

Solid and triangle curves in Fig. 1 show XPS (Mg $\mathrm{K}_{\alpha}$ excited) spectra for $\mathrm{C} 1 s$ core level of the sample B$\mathrm{C}(001) \mathrm{H}$ at an area excluding and including the graphite patch on the surface, respectively. The dotted circles in the inset of Fig. 1 show schematically X-ray beam areas excluding (arrow D) and including (arrow D+G) the graphite patch on the surface. The dotted curve in Fig. 1 shows $\mathrm{C} 1 s$ XPS spectrum of the reference graphite near the sample. It is seen in Fig. 1 that $\mathrm{C} 1 s$ levels for B$\mathrm{C}(001) \mathrm{H}$ and the reference graphite are located very close to each other. Therefore, C $1 s$ XPS peak of B-C(001)H for the area including the graphite patch appears very similar to that of $\mathrm{B}-\mathrm{C}(001) \mathrm{H}$ alone. This, in turn, means very small energy shift at the junction between graphite and $\mathrm{B}-\mathrm{C}(001) \mathrm{H}$ sample surface. In order to assess quantitative measure of the energy shift, we have evaluated C $1 s$ component due to the graphite patch in Fig. 1 as small circle curve. The evaluation was performed in such a way that diamond and graphite components in the curve 'D+G' have same spectral profiles as those of diamond and graphite. The intensity of resulting diamond component is $\sim 77 \%$ of that of ' $\mathrm{D}$ ' curve with no energy shift and the intensity of resulting graphite component is $\sim 23 \%$ of that of graphite curve with an energy shift of $\sim 0.1 \mathrm{eV}$ toward the higher kinetic energy side. If a built-up potential were present at the junction between $\mathrm{B}-\mathrm{C}(001) \mathrm{H}$ and graphite during the XPS measurement, a non-marginal energy shift of graphite patch component would have resulted.

Figure 2 shows a current $\left(I_{0}\right)$ vs. voltage $\left(V_{0}\right)$ curve measured in-situ on the hP-C(111)O sample. Schematics of the measurement is shown as an inset of Fig. 2. We used a movable $\mathrm{W}$-wire $(0.2 \mathrm{~mm}$ in diameter $)$ electrode probe, whose tip was covered with graphite paste, similar to a two-point probe used previously [7]. It is seen in Fig. 2 that the electric contact between hP-C(111)O surface and graphite electrodes is good enough for XPS and SES measurements without any potential built-up although the electric contact may not be a perfect ohmic contact. For the hP-C(111)H sample, we can expect the similar junction properties since the graphite electrode was pasted on the hP-C(111)H sample initially and then annealed in air to make the sample hP-C(111)O as described in Section 2.

\section{B. C $1 s$ XPS peaks and SES spectra for all the samples}

Figure 3 shows comparison of $\mathrm{C} 1 s$ XPS peaks among the samples of B-C(001)H, hP-C(111)H and hP-C(111)O. It is seen in Fig. 3 that $\mathrm{C} 1 s$ peak positions for $\mathrm{hP}$ $\mathrm{C}(111) \mathrm{H}$ and $\mathrm{hP}-\mathrm{C}(111) \mathrm{O}$ are shifted by $\sim 2.1$ and $\sim 1.8$ $\mathrm{eV}$ from that for $\mathrm{B}-\mathrm{C}(001) \mathrm{H}$, respectively. The full width at half maximum (FWHM) of $\mathrm{C} 1 s$ peaks are evaluated to be 2.1 and $2.3 \mathrm{eV}$ for hP-C(111) H and hP-C(111)O, respectively. FWHM of $\mathrm{C} 1 s$ XPS peak for $\mathrm{B}-\mathrm{C}(001) \mathrm{H}$ is evaluated to be $1.1 \mathrm{eV}$. This comparison illuminates a large difference in surface energy band diagram between the highly $\mathrm{P}$-doped samples and the B-doped $p$-type sample.

Figure 4 shows comparison of intensity-normalized SES spectra among the samples of $\mathrm{B}-\mathrm{C}(001) \mathrm{H}, \mathrm{hP}-\mathrm{C}(111) \mathrm{H}$ and hP-C(111)O. For B-C(001)H sample, He I/II excited SES spectrum is also displayed. It can be seen in Fig.4 that the cutoff energies (as indicated by arrows) of Xray excited SES peaks are 4.4,3.8 and $3.9 \pm 0.1 \mathrm{eV}$ for $\mathrm{B}-\mathrm{C}(001) \mathrm{H}, \mathrm{hP}-\mathrm{C}(111) \mathrm{H}$ and $\mathrm{hP}-\mathrm{C}(111) \mathrm{O}$, respectively. The cutoff energies are defined as onsets of SES intensities since the low-energy slopes of SES peaks are results of the density of unoccupied states rather than an energy resolution of the spectrometer. He I/II excited SES spectrum for B-C(001)H showed another cutoff feature as marked by an arrow at $4.8 \mathrm{eV}$. The cutoff feature at 4.4 $\mathrm{eV}$ can be ascribed to the bottom of unoccupied states of hydrogen terminated diamond (001) surface and the second cutoff feature at $4.8 \mathrm{eV}$ can be ascribed to the bottom of conduction band as already ascribed for H-terminated diamond (001) [9]. It is to be noted here that a double- 

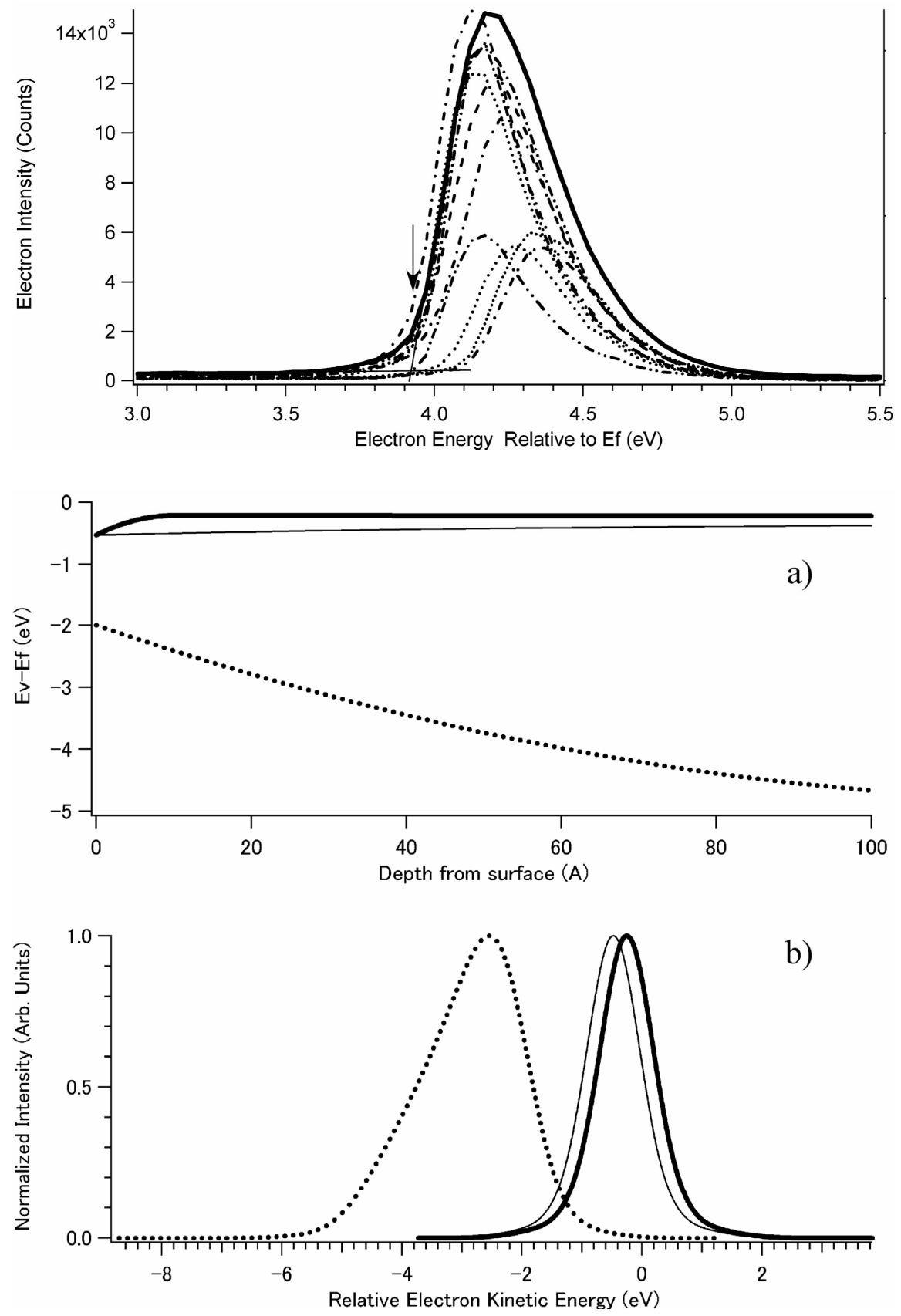

FIG. 6: SES spectra as measured by IS-PEEM for hPC(111)O. Dotted curves are ten SES spectra measured from typical ten areas of $\sim 10 \mu \mathrm{m}$ in diameter in Fig. 4. The solid curve is a normalized sum of the ten micro-SES spectra. peak structure in SES for a boron-doped $\mathrm{C}(001) \mathrm{H}$ sample as seen in Fig. 10 of Ref. [9] could be an artifact due to detector saturation. A similar double-peak structure was found in this study when a large spectrometer entrance slit was used with a counting rate exceeding $\sim 10^{6}$ cts/s. Valence band photoelectron spectrum as excited by He II resonance light (not shown) for the $\mathrm{B}-\mathrm{C}(001) \mathrm{H}$ showed that the valence band top is located at $40.2 \pm 0.1$ $\mathrm{eV}$ with reference to $E_{F}$. By reducing photon energy $(40.82 \mathrm{eV})$ of He II resonance light, the valence band top is located at $-0.65 \pm 0.1 \mathrm{eV}$, thus the conduction band bottom at $4.9 \pm 0.1 \mathrm{eV}$. This proves the assignment of the feature at $4.8 \mathrm{eV}$ being the conduction band bottom of $\mathrm{H}$ terminated $\mathrm{C}(001)$ surface reasonable. X-ray excited SES spectrum for B-C(001)H surface in Fig. 4 shows a strong peak above the second cutoff feature of $4.8 \mathrm{eV}$, which indicates that secondary electrons along off-normal directions were mostly detected due to geometrical configuration for
X-ray excited SES.

X-ray excited SES spectra for $\mathrm{hP}-\mathrm{C}(111) \mathrm{H}$ and $\mathrm{hP}$ $\mathrm{C}(111) \mathrm{O}$ in Fig. 4 show no characteristic features other than the cutoff features, thus the surface energy band diagrams for these samples can be evaluated only with comparison to that of $\mathrm{B}-\mathrm{C}(001) \mathrm{H}$ through $\mathrm{C} 1 s$ XPS and SES spectra.

\section{PEEM image and micro-SES spectra for the sample hP-C(111)O}

Figure 5 shows a combined PEEM image of the sample hP-C(111)O as excited by the Hg-lamp. The sample surface looks rather inhomogeneous with one large defect/particle and many small dark spots. Otherwise, most parts of the sample surface are bright and rather uniform. Ten SES spectra measured from typical ten areas of $\sim 10$ 


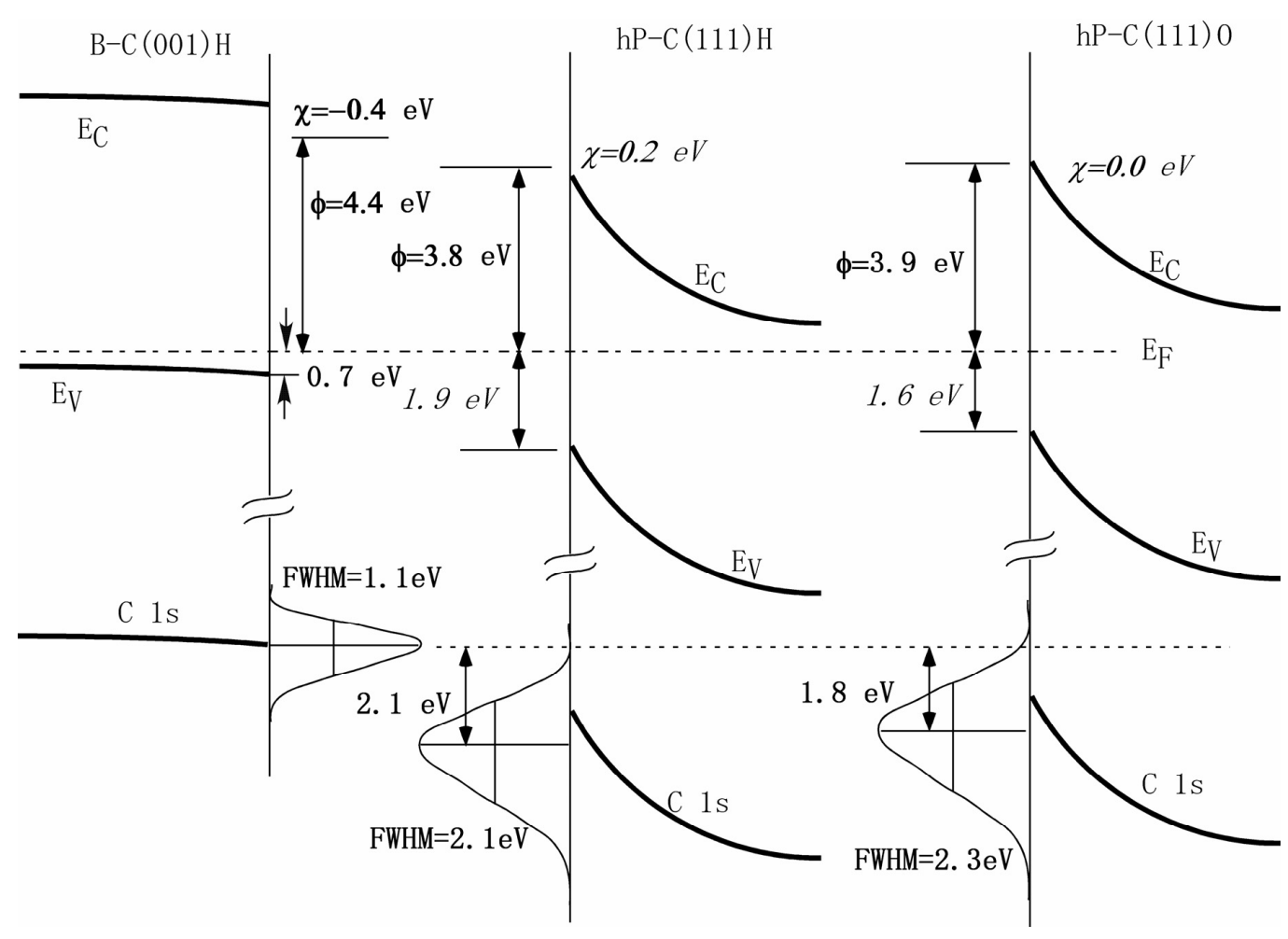

FIG. 8: A schematic of the surface energy band diagrams for the three samples of B-C(001)H, hP-C(111)H and hP-C(111)O. Critical values as determined in the present work are given.

$\mu \mathrm{m}$ in diameter in Fig. 5 are shown as dotted curves in Fig. 6. They show deviation of about $0.25 \mathrm{eV}$ in peak positions and cutoff energies. This certainly shows that the sample surface is not uniform in energy band structure but is deviated by an amount of $\sim \pm 0.13 \mathrm{eV}$. However, this also shows that the large FWHM of $2.3 \mathrm{eV}$ in $\mathrm{C} 1 s$ XPS peak in Fig. 3 is not much affected by the nonuniformity of the sample surface. It is also to be noted that the FWHM in C $1 s$ XPS peak may be affected by a possible maximum amount of $\sim \pm 0.13 \mathrm{eV}$ if the surface non-uniformity is related solely to surface Fermi-pinning position. The ten SES spectra are summed over and normalized as a solid curve in Fig. 6. The summed SES curve shows a cutoff energy (as marked by an arrow) at $3.9 \sim 0.05 \mathrm{eV}$, which agrees with that of macroscopic value in Fig. 4.

\section{ANALYSIS OF SURFACE ENERGY BAND DIAGRAM}

Using critical values in energy band as found in experiment in Section 3, we have simulated surface energy band diagrams for the reference sample $\mathrm{B}-\mathrm{C}(001) \mathrm{H}$ and for the highly $\mathrm{P}$-doped samples of $\mathrm{hP}-\mathrm{C}(111) \mathrm{H}$ and $\mathrm{hP}-\mathrm{C}(111) \mathrm{O}$. The simulation was performed using a $1 \mathrm{D}$ band diagram calculator (1D Poisson) [10]. The acceptor ionization energy for $\mathrm{B}$ was set to $0.32 \mathrm{eV}$ and the donor ionization energy for $\mathrm{P}$ was set to $0.6 \mathrm{eV}$. The simulated surface energy band diagrams were used to further simulate $\mathrm{C}$ $1 s$ XPS peaks of the three samples. In the simulation of $\mathrm{C} 1 s$ XPS peaks, we need two important parameters that are an electron mean free path and an intrinsic $\mathrm{C} 1 \mathrm{~s}$ peak line shape. The electron mean free path in diamond is rather problematic in electron spectroscopy [11]. We used $2.8 \mathrm{~nm}$ as a reasonable choice in accordance with a value used in a similar analysis [9]. As a reasonable choice for $\mathrm{C} 1 s$ peak line shape, we used a Voigt function, i.e., a Lorentzian function convoluted with a Gaussian function. A HWHM of $0.3 \mathrm{eV}$ for the Lorentzian was used to be consistent with life time broadening [12] of $\mathrm{C} 1 s$ peak and a FWHM of $0.9 \mathrm{eV}$ for the Gaussian was used to be consistent with the energy resolution of $\mathrm{Mg} \mathrm{K}_{\alpha}$ excited XPS peaks.

As noted in Section 2, the sheet resistance of the second $\mathrm{B}-\mathrm{C}(001) \mathrm{H}$ sample as loaded in the CLAM4 chamber was $\sim 1 \mathrm{M} \Omega$ /square and that after annealing in vacuum at $650^{\circ} \mathrm{C}$ was $\sim 1.9 \mathrm{M} \Omega$ /square. Although sheet resistance of the first reference sample of $\mathrm{B}-\mathrm{C}(001) \mathrm{H}$ was not measured, a similar behavior can be expected. It is also expected that a surface conductive layer exists before the invacuum annealing and vanishes after in-vacuum annealing at $650^{\circ} \mathrm{C}$ [7]. Sheet resistance values are other factors that are to be fitted by 1D Poisson. Simulation of surface energy band with the presence of surface conductive layer was performed in a similar manner as before [13]. Simulation of surface energy band with the presence of surface conductive layer based on the so-called surface transfer doping model [14] would not be appropriate since the valence band maximum is found to be located $0.65 \pm 0.1 \mathrm{eV}$ below $E_{F}$ at the surface.

Thick and thin solid lines in Fig. 7a) show the simulated valence band top $\left(E_{V}\right)$ position for the reference sample with and without surface conductive layer, re- 
spectively. The valence band top lies at $0.65 \mathrm{eV}$ below the Fermi level $\left(E_{F}\right)$ at the surface as found experimentally. Sheet resistances are consisted with those experimental values. This gave a bulk boron concentration of $\sim 1 \times 10^{18} / \mathrm{cm}^{3}$ and a concentration of possible shallow level acceptors of $\sim 2.7 \times 10^{20} / \mathrm{cm}^{3}$ within a depth of $1 \mathrm{~nm}$. The resulting valence band diagrams show two extreme cases and the actual band diagram is expected somewhere in between. It is also to be noted that bulk valence band top is located $\sim 0.25 \mathrm{eV}$ below the $E_{F}$.

Thick and thin solid curves in Fig. 7b) show simulated C $1 s$ XPS peaks for the reference sample with and without surface conductive layer, respectively. Relative electron kinetic energy is shown in such a way that the origin corresponds to the case when the valence band top coincides with $E_{F}$. It is seen in Fig. $7 \mathrm{~b}$ ) that $\mathrm{C} 1 s \mathrm{XPS}$ peak is assigned within an error of $\pm 0.15 \mathrm{eV}$. It is to be noted that an asymmetrical shape of the experimental $\mathrm{C}$ $1 s$ XPS peak in Fig. 3 is due to a surface hydrocarbon component at a lower kinetic energy side [15], which is not taken into account in the simulated curve.

Dotted curves in Fig. 7a) and 7b) show a typical example of simulated $E_{V}$ position and simulated C $1 s$ XPS peak, respectively, for a highly P-doped diamond. In this case, $E_{V}$ at the surface lies at $2.0 \mathrm{eV}$ below $E_{F}$, whose precise position should be re-adjusted from $\mathrm{C} 1 s$ XPS peak positions for $\mathrm{hP}-\mathrm{C}(111) \mathrm{H}$ and hP-C(111)O. Phosphorous concentration was adjusted to have a FWHM of C $1 s$ XPS peak at $2.0 \mathrm{eV}$, which is a few tenth of an $\mathrm{eV}$ smaller than those found in experiments as in Fig. 3. Micro-SES spectra as in Fig. 6 showed that possible surface non-uniformity causes an additional broadening of $\sim 0.2 \mathrm{eV}$. A donor $(\mathrm{P})$ concentration as determined was $\sim 1 \times 10^{19} / \mathrm{cm}^{3}$, which is consistent with a result $\left(\sim 9 \times 10^{19} / \mathrm{cm}^{3}\right)$ of SIMS analysis of highly P-doped samples synthesized in a similar way [5] if a large amount of compensation ratio of $\sim 8 / 9$ is taken into account.

Figure 8 schematically shows a summary of the surface energy band diagrams and the electron affinities for the three samples of $\mathrm{B}-\mathrm{C}(001) \mathrm{H}, \mathrm{hP}-\mathrm{C}(111) \mathrm{H}$ and $\mathrm{hP}-$ $\mathrm{C}(111)$ O. In Fig. 8, thick solid lines as marked $E_{C}, E_{V}$ and $\mathrm{C} 1 s$ are the conduction band bottom, the valence band top and $\mathrm{C} 1 s$ core level, respectively. The values in bold are those determined experimentally and the values in italics are estimated values from the simulation of surface energy bands and $\mathrm{C} 1 s$ XPS peaks. The value $\chi=-0.4 \mathrm{eV}$ for $\mathrm{B}-\mathrm{C}(001) \mathrm{H}$ is an lower bound for the electron affinity as determined in SES, which is a little smaller than that determined for a lower concentration $\left(1 \times 10^{16} / \mathrm{cm}^{3}\right)$ B-dope $\mathrm{C}(001) \mathrm{H}$ sample [9]. This difference may be related to the difference in dopant concentration or in surface cleanness condition. Since we have an error of $\pm 0.15 \mathrm{eV}$ in the determination of $\mathrm{C} 1 s$ peak for $\mathrm{B}-\mathrm{C}(001) \mathrm{H}$ due to possible surface conductive layer, the electron affinity $\chi$ values for hP-C(111)H and hP-C(111)O may vary accordingly. The $\chi$ value for $\mathrm{hP}-\mathrm{C}(111) \mathrm{H}$ is positive but is close to zero and $\chi$ value for $\mathrm{hP}-\mathrm{C}(111) \mathrm{O}$ is close to zero and can be negative. It is certain that the energy bands for the highly P-doped samples bent upward toward surface by an amount of $\sim 3 \mathrm{eV}$, thus a strong electric field is needed to extract carrier electrons from the conduction band into vacuum.

\section{CONCLUSIONS}

The surface energy band diagrams and the electron affinities of both hydrogen-terminated and oxygenterminated highly phosphorous-doped single crystal diamonds (hP-C(111)H and hP-C(111)O), which are substrates for good field emission emitters, have been studied by ultraviolet photoelectron spectroscopy, secondary electron spectroscopy, X-ray photoelectron spectroscopy and photo-emission electron micro-spectroscopy. A hydrogenterminated boron-doped diamond sample (B-C(001)H) was also studied as a reference of surface energy band diagram. The electron affinity of the sample hP-C(111)H is $\sim 0.2 \pm 0.15 \mathrm{eV}$, thus close to zero. The electron affinity of the sample $\mathrm{hP}-\mathrm{C}(111) \mathrm{O}$ is $\sim 0.0 \pm 0.15 \mathrm{eV}$, thus can be negative. However, the surface energy bands for the two highly P-doped samples are found to have large amounts ( $\sim 3 \mathrm{eV})$ of upward bending toward surface. This indicates that a strong electric field is needed to extract carrier electrons from the conduction band into vacuum, which may makes the highly P-doped diamonds not to be much suited for field emitters.

\section{Acknowledgments}

This work is supported in part from Advanced Diamond Device Project administered by NEDO, Japan. The authors are grateful to Prof. H. Kawarada and Dr. H. Umezawa for the supply of the reference samples of B$\mathrm{C}(001) \mathrm{H}$.
[1] S. Koizumi, K. Watanabe, M. Hasegawa and H. Kanda, Science 292, 1899 (2001).

[2] M. Katagiri, J. Isoya, S. Koizumi and H. Kanda, Appl. Phys. Lett. 85, 6365 (2004).

[3] C. Tavares, S. Koizumi and H. Kanda, Phys. Stat. Solid A 202, 2129 (2005).

[4] S. Koizumi, Phys. Stat. Solid (a) 172, 71 (1999).

[5] As a review, A. Namba, N. Tatsumi, Y. Yamamoto, Y. Nishibayashi and T. Imai, SEI Technical Review 166, 38 (2005) (in Japanese).

[6] Y. Nishibayashi, Y. Ando, H. Furuta, K. Kobashi, K. Me- guro, T. Imai, and K. Oura, New Diamond and Frontier Carbon Technol. 13, 19 (2003).

[7] S. Kono, M. Shiraishi, T. Goto, T. Abukawa, M. Tachiki and H. Kawarada, Diamond and Relat. Mater. 14, 459 (2005).

[8] S. Kono, T. Goto, T. Abukawa, Y. Takakuwa, K. Sato, H. Yagi, T. Ito, Diamond and Relat. Mater. 10, 48 (2001).

[9] L. Diederich, O. M. Kuttel, P. Aebi, and L. Schlapbach, Surf. Sci. 418, 219 (1998).

[10] G. L. Snider, I.-H. Tan and E. L. Hu, J. Appl. Phys. 68, 2849 (1990). 
[11] S. Tanuma, C. J. Powell and D. R. Penn, Surf. Interface Anal. 36, 1 (2004).

[12] F. Sette, G. K. Wertheim, Y. Ma, G. Meigs, S. Modesti and C. T. Chen, Phys. Rev. B 41, 9766 (1990).

[13] S. Kono and Y. Koide, Jpn. J. Appl. Phys. 44, 8378 (2005).
[14] P. Strobel, M. Riedel, J. Ristein and L. Ley, Nature 430, 439 (2004), and references therein.

[15] R. Graupner, F. Maier, J. Risein and L. Ley, Phys. Rev. B 57, 12397 (1998). 